\title{
Simple Devices to Facilitate the Immunization of Horse for Antivenom/Antitoxin Production
}

Akarin Intaramat ${ }^{1}$, Pachern Puthsorn ${ }^{2}$, Sansaneeyaporn Kittikajhon ${ }^{2}$, Hathaichanok Eamrod ${ }^{2}$ and Kavi Ratanabanangkoon ${ }^{1,2^{*}}$

${ }^{1}$ Chulabhorn Research Institute and Chulabhorn Graduate Institute, Bangkok, Thailand

${ }^{2}$ The Government Pharmaceutical Organization of Thailand, Thailaland

"Corresponding author: Kavi Ratanabanangkoon, PhD, Laboratory of Immunology, Chulabhorn Research Institute and Chulabhorn Graduate Institute, Vipavadee Rangsit Hwy, Laksi, Bangkok 10210,Thailand, Tel: (662) 574-0622; E-mail: kavi.rtn@mahidol.ac.th

Rec date: May 30, 2015; Acc date: Aug 10, 2015; Pub date: Aug 12, 2015

Copyright: @ 2015 Ratanabanangkoon K, et al. This is an open-access article distributed under the terms of the Creative Commons Attribution License, which permits unrestricted use, distribution, and reproduction in any medium, provided the original author and source are credited.

\begin{abstract}
A multi-dose injector to facilitate the immunization of horse by the 'low dose, low volume multi-site' protocol in antivenom/antitoxin production is described. With the device, 20 subcutaneous injections of about $0.1 \mathrm{ml}$ of CFA emulsified immunogen around the horse's neck could be easily accomplished within 3 minutes without any stress to the animal. Also described is an apron to prevent blisters and infection at the injection sites caused by the horse rubbing the neck against stable and soil. These devices have been found to be very useful to the investigator and in reducing the pain and suffering of the horses during immunization for the production high potency antivenom/ antitoxin.
\end{abstract}

Keywords: Horse immunization; Antivenom/Antitoxin production; Low volume multi-site immunization; Multi-dose injector

\section{Introduction}

Production of therapeutic antivenoms or antitoxins in horses has been carried out for decades but it is still plagued with problems and improvements are needed [1]. One of the problems is the poor antibody response of the horse against snake venoms. Horses are sensitive to the Complete Freund adjuvant (CFA) used in the immunization, the use of this adjuvant was subsequently discouraged and bentonite was used instead [2]. However, with bentonite as an adjuvant the antibody response was very poor [3]. In the past few years, immunization using CFA has been reintroduced using a 'low dose, low volume multi-site' immunization protocol with good results in terms of high antibody titer, short time to reach maximum titer and high avidity antibody with minimal local tissue necrosis [4-8]. However, some problems remain in this immunization protocol. The 15-20 subcutaneous injections with low volume (ca 0.05-0.1 ml) at each site in the neck areas are difficult to carry out and cause undue suffering to the horse.

Furthermore, after immunization the horses often rub the injected areas with the stable wall or on the soil, resulting in blisters and infection of the injection sites.

We describe here a multi-dose injection device to facilitate immunization, and an apron designed to cover the injection areas. With this equipment, the immunization can be easily and rapidly achieved with no stress on the horse, while the use of apron prevents any blistering and infection at the injection site.

\section{Materials and Methods}

The injection device to facilitate immunization, a multi-dose injector with varying dose volume $(0.1-0.3 \mathrm{ml})$ was used (Ultimate BMV applicator, Instrument Supplies, New Zealand). For immunization of a group of 5 horses, an immunogen prepared in complete or incomplete Freund adjuvant $(20 \mathrm{ml})$ was filled in a $20 \mathrm{ml}$ capacity glass serum bottle using a protocol described previously by WHO Guidelines [9]. The injector was fitted with a $38 \mathrm{~mm}$ no. 21 gauge disposable needle. The needle cover with its end cut off was attached so that only $2-3 \mathrm{~mm}$ of the needle tip was exposed and penetrated the horse skin (Figure 1).

An apron was designed to cover the neck area to prevent blister formation and infection resulting from the horse rubbing the injection sites against the stable and/or soil (Figure 2). The apron was made of canvass with soft clean towel attached and lined inside in such a way that the towel could be changed daily.

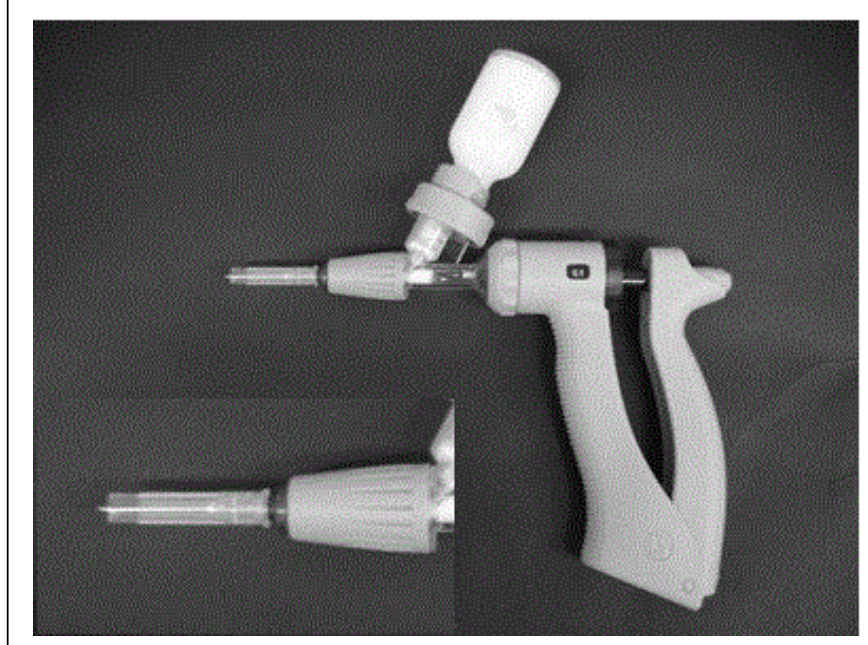

Figure 1: The multi-dose injector with CFA emulsified immunogen in a bottle and a piece of plastic tube.In set, the needle is fitted with the plastic tube to control the depth in injection. 
Citation: Intaramat A, Puthsorn P, Kittikajhon S, Eamrod H, Ratanabanangkoon K, (2015) Simple Devices to Facilitate the Immunization of Horse for Antivenom/Antitoxin Production. J Veterinar Sci Technol 6: 249. doi:10.4172/2157-7579.1000249

Page 2 of 2

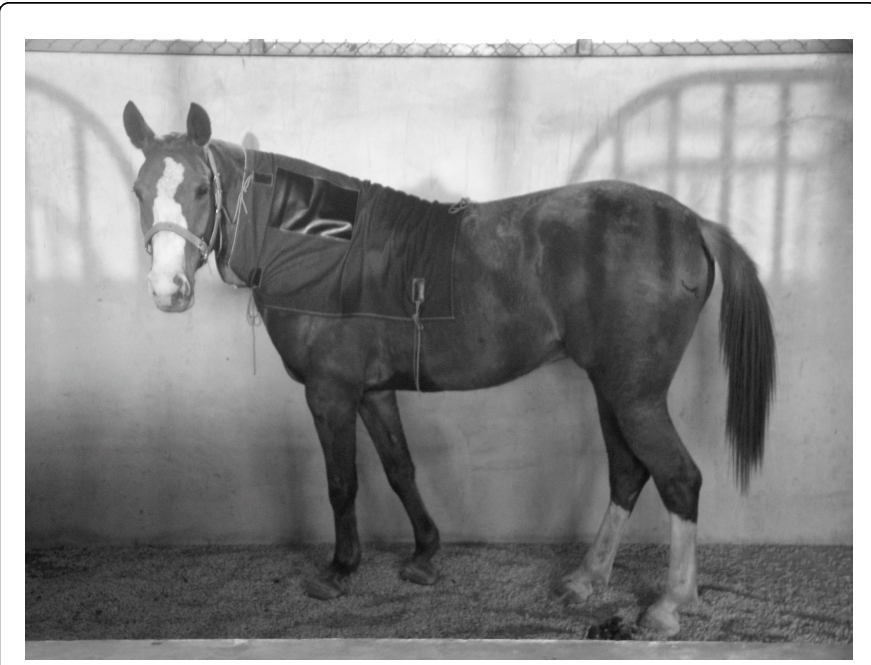

Figure 2: An apron used to prevent blisters and infection at the injection sites around the horse's neck. Elastic bands were used to tie the apron in place and to allow for unrestricted movement of the horse.

\section{Results and Discussion}

The injector with the multi-dose injector, immunization could be performed by injection and expulsion of the immunogen almost simultaneously in one single step. Ten such injections on one side of the horse's neck could be done in less than $1 \mathrm{~min}$ and the horse remained calm and did not show any sign of distress at all. Twenty such injections on both sides of the neck could be accomplished in less than 3 minutes. With the 21 gauge needle used, there was no observable leakage of immunogen from the horse skin after injection. When the volume of the injector was set at $100 \mu \mathrm{l}$, the volume of the viscous liquid (density $0.954 \mathrm{~g} / \mathrm{ml}$ ) of CFA emulsified immunogen of $84.79 \pm 1.24 \mu \mathrm{l}$ was delivered.

The injection device, without the immunogen, could be sterilized by fuming under hydrogen peroxide plasma (Sterrar ${ }^{\oplus}$, Advanced Sterilization Products, Ethicon, Inc.) but not by autoclave. The barrel could not be washed with organic solvents to remove the oil adjuvant without harming the plastic/rubber O-rings. The barrel cost about US \$4.5. The described multi-dose injection costs about US \$ 12 .

The apron was worn on the horse at all times in the stable and in the field and was changed and cleaned daily. The horses did not show any sign of rejection of the apron.

With this apron, the injection sites of all 10 horses, 5 immunized with CFA emulsified Calloselasma rhodostoma venom and 5 with
CFA emulsified tetanus toxoid, showed swelling during the first 2 weeks and some horses showed $102^{\circ} \mathrm{C}$ fever only on the 2 nd-3rd day after immunization. No blister or infection was observed at any of the 134 injection sites during the 4 weeks post immunization. Each apron can easily be produced locally and costs about US \$ 10 in Thailand.

The antibody response. After 4 immunizations over 8 week periods [4], the antibody responses against either C. rhodostoma venom or tetanus toxoid were found to be rapid and high, similar to those observed with N. kaouthia venom (data not shown).

\section{Conflict of interest}

None

\section{Acknowledgements}

The authors thank Dr Witit Artavakun, Mr. Somchai Srichainak, Mr. Sit Thirapakpoomanunt for valuable suggestions.

\section{References}

1. Williams DJ, Gutiérrez JM, Calvete JJ, Wüster W, Ratanabanangkoon K, et al. (2011) Ending the drought: new strategies for improving the flow of affordable, effective antivenoms in Asia and Africa. J Proteomics 74: 1735-1767.

2. Christensen PA (1979) Production and standardization of antivenom, In Handbook of Experimental Pharmacology, Lee CY (Ed) Springer, Berlin, 52: 825-846.

3. Sunthornandh P, Ratanabanangkoon K (1994) A comparative study of three vehicles on antibody responses against elapid snake neurotoxin immunogens. Toxicon 32: 561-571.

4. Pratanaphon R, Akesowan S, Khow O, Sriprapat S, Ratanabanangkoon K (1997) Production of highly potent horse antivenom against the Thai cobra (Naja kaouthia) Vaccine 15: 1523-1538.

5. Chotwiwatthanakun C, Pratanaphon R, Akesowan S, Sriprapat S, Ratanabanangkoon K (2001) Production of potent polyvalent antivenom against three elapid venoms using a low dose, low volume, multi-site immunization protocol. Toxicon 39: 1487-1494.

6. Sriprapat S, Aeksowan S, Sapsutthipas S, Chotwiwatthanakun C, Suttijitpaisal P, et al. (2003) The impact of a low dose, low volume, multisite immunization on the production of therapeutic antivenoms in Thailand. Toxicon 41: 57-64.

7. El-Kady EM, Ibrahim NM, Wahby AF (2009) Assessment of the antiNaja haje antibodies elicited in a low dose multi-site immunization protocol. Toxicon 54: 450-459.

8. Sapsutthipas S, Leong PK, Akesowan S, Pratanaphon R, Tan NH, et al. (2015) Effective equine immunization protocol for production of potent poly-specific antisera against Calloselasma rhodostoma, Cryptelytrops albolabris and Daboia siamensis. PLoS Negl Trop Dis 9: e0003609.

9. World Health Organization Guidelines for the Production (2010) Control and Regulation of Snake Antivenom Immunoglobulins, WHO, Geneva. 